\title{
Interaction of a C-terminal Truncated Hepatitis C Virus Core Protein with Plasmid DNA Vaccine Leads to in vitro Assembly of Heterogeneous Virus-like Particles
}

\author{
${ }^{1}$ Nelson Acosta-Rivero, ${ }^{1}$ Joanna Poutou, ${ }^{1}$ Alexis Mussachio, ${ }^{1}$ Viviana Falcon, ${ }^{1}$ Yaraima Aguilera, \\ ${ }^{1}$ Armando Rodriguez, ${ }^{1}$ Angel Perez, ${ }^{1}$ Julio C. Aguilar, ${ }^{1}$ Maria C de la Rosa, ${ }^{1}$ Felix Alvarez \\ ${ }^{1}$ Juan Morales-Grillo, ${ }^{2}$ Juan Kouri and ${ }^{1}$ Santiago Dueñas-Carrera \\ ${ }^{1}$ Biomedical Research Division, Center for Genetic Engineering and Biotechnology \\ P.O. Box 6162, C.P. 10600, C. Habana, Cuba \\ ${ }^{2}$ Centro de Investigación y de Estudios Avanzados (CINVESTAV-IPN), México City, México
}

\begin{abstract}
Recently, it has been shown that $\mathrm{HCV}$ core proteins ( $\mathrm{HCcAg}$ ) with C-terminal deletions assemble in vitro into virus-like particles (VLPs) in the presence of structured RNA molecules. Results presented in this work showed that a truncated $\mathrm{HCcAg}$ variant covering the first 120 aa (HCcAg.120) with a 32 aa N-terminal fusion peptide (6xHistag-Xpress ${ }^{\mathrm{TM}}$ epitope) interacts with plasmid DNA vaccine. Interestingly, the buoyant density of VLPs containing $\mathrm{HCcAg} .120$ in $\mathrm{CsCl}$ gradients changed from $1.15-1,17 \mathrm{~g} \mathrm{~mL}^{-1}$ to $1.30-1.34 \mathrm{~g} \mathrm{~mL}^{-1}$ after addition of plasmid DNA to assembly reactions. In addition, a delay in electrophoretic mobility of HCcAg.120-plasmid samples on agarose gels was observed indicating a direct interaction between VLPs and nucleic acids. Remarkably, addition of either plasmid DNA or tRNA to assembly reactions leaded to heterogeneous and larger VLPs formation than those observed in HCcAg.120 assembly reactions. VLPs containing HCcAg.120 induced a specific IgG antibodies in mice that reacted with hepatocytes from $\mathrm{HCV}$-infected patients. VLPs obtained in this work would be important to elucidate the mechanisms behind the ability of $\mathrm{HCcAg}$ to assemble into a nucleocapsid structure. Besides, the capacity of particles containing HCcAg.120 to interact with nucleic acids could be used in the development of DNA vaccines and viral vectors based on these particles.
\end{abstract}

Key words: E. coli, hepatitis C, core antigen, virus like particles

\section{INTRODUCTION}

Hepatitis $\mathrm{C}$ virus (HCV) is a major worldwide health problem. $\mathrm{HCV}$ is a member of the Hepacivirus genus of the Flaviviridae family ${ }^{[1]}$. The genome is a single-stranded positive-sense RNA of approximately $9600 \mathrm{nt}$ and encodes a polyprotein with a single open reading frame (ORF) of $3008-3033$ aa which is processed by host and viral proteases ${ }^{[2]}$. The structural proteins (core, E1, E2 and potentially p7) precede the nonstructural proteins (NS2, 3, 4A, 4B, 5A and 5B) in the polyprotein. The biogenesis of $\mathrm{HCV}$ core protein $(\mathrm{HCcAg})$ is dependent on the interaction of the signal sequence of nascent polypeptide with the endoplasmic reticulum (ER) membrane ${ }^{[3]}$. Although it has been suggested that $\mathrm{HCcAg}$ function to encapsidate the viral genome within a nucleocapsid particle, little is known about the in vivo assembly pathway or structure of the HCV nucleocapsid and virion ${ }^{[4]}$.

Since virion nucleocapsid formation involves multimerization of $\mathrm{HCcAg}$ and its interaction with viral RNA, these characteristics of $\mathrm{HCcAg}$ have been extensively studied and specific functions mapped to discrete portions of the molecule ${ }^{[5-8]}$. Difficulties in obtaining mature $\mathrm{HCcAg}$ have required that core proteins with $\mathrm{C}$-terminal deletions be used for in vitro functional studies ${ }^{[9-11]}$. In addition, these $\mathrm{HCcAg}$ variants self-assemble into nucleocapsids in cell-free assays in the presence of structured nucleic acids ${ }^{[9,11]}$. However, there are still many questions open about the assembly properties of $\mathrm{HCcAg}$.

Previously, it has been reported that a C-terminal truncated $\mathrm{HCcAg}$ variant (covering aa 1-120) with a 6xHis tag fusion at N-terminus (HCcAg.120) assembled into virus-like particles (VLPs) when expressed in recombinant Escherichia coli cells $^{[10]}$. On the other hand, a previous study had demonstrated that a DNA vaccine containing the structural region of $\mathrm{HCV}$ (pIDKE2 plasmid) induces a strong humoral and cellular immune response in various animal models ${ }^{[12,13]}$. Since particulate antigens have been proposed as potential carriers for DNA vaccines ${ }^{[14]}$, the capacity of HCcAg.120 to interact with pIDKE2 plasmid and to assemble into VLPs in vitro was investigated in this study.

Corresponding Author: Nelson Acosta-Rivero, Hepatitis C Department, Center for Genetic Engineering and Biotechnology, Ave. 31 e/ 158 and 190, P. O. Box 6162, C.P. 10 600, La Habana, Cuba, Tel: (53 7) 271 4764, Fax: (53 7) 2714764 


\section{MATERIALS AND METHODS}

Plasmid, antibodies and strain: The pIDKE2 plasmid containing the structural region of $\mathrm{HCV}$ ( $\mathrm{HCcAg}, \mathrm{E} 1$ and E2) has been previously described ${ }^{[12]}$. It was used in protein-nucleic acid interaction studies. A mouse monoclonal antibody against the residues 5-35 of HCcAg (mAb SS-HepC.1) was used to detect HCcAg. $120^{[15]}$. Anti-HCcAg.120 IgG antibodies obtained in mice immunized with VLPs containing $\mathrm{HCcAg} .120$ were used in immunoelectron and immunofluorescence microscopy analysis. The BL21(DE3)pLysS strain was used to express HCcAg.120 as previously reported ${ }^{[10]}$.

HCcAg.120 purification from recombinant Escherichia coli cells: HCcAg.120 was semipurified under denaturing conditions with purity higher than $85 \%$ as previously described ${ }^{[10]}$. Later on, $\mathrm{HCcAg} .120$ in denaturing buffer (DB) $(8 \mathrm{M}$ urea, $20 \mathrm{Mm}$ Tris-HCL $\mathrm{pH} 8,250 \mathrm{mM} \mathrm{NaCl}$ ) was applied to reverse-phase (RP) high-pressure liquid chromatography (HPLC) C8 column (either $4.6 \times 150 \mathrm{~mm}$ or $10 \times 250 \mathrm{~mm}$ ) (Vydac HPLC columns, USA) as previously described ${ }^{[9,16]}$. It was eluted using a linear gradient of acetonitrile ( $\mathrm{AcN})$ in $5 \%$ trifluoroacetic acid (TFA) at $1 \mathrm{~mL} \mathrm{~min}^{-1}$ flow rate. The major eluted fraction containing $\mathrm{HCcAg} .120$ was dialyzed overnight at $4^{\circ} \mathrm{C}$ against TNGU buffer (20 mM Tris [pH 7.0], $100 \mathrm{mM} \mathrm{NaCl}, 1 \%$ Glycerol, $2 \mathrm{M}$ Urea). Later on, HCcAg. 120 was concentrated by $30 \%$ ammonium sulfate precipitation and resuspended at $2 \mathrm{mg} \mathrm{mL}^{-1}$ in TNGU buffer. Finally, HCcAg.120 was dialyzed overnight at $4^{\circ} \mathrm{C}$ against refolding buffer (RB) (20 mM Tris [pH 7.0], $100 \mathrm{mM} \mathrm{NaCl}, 1 \%$ Glycerol). Renatured HCcAg.120 sample (after a 16,000 g spin at $4^{\circ} \mathrm{C}$ for $15 \mathrm{~min}$ ) was tested in three percent agarose gels prepared in $20 \mathrm{mM}$ Tris-acetate-0.5 mM EDTA (TA) buffer and stained with TA buffer containing $10 \mathrm{ug}$ $\mathrm{mL}^{-1}$ of ethidium bromide. HCcAg. 120 was purified as a single protein with purity higher than $90 \%$.

HCcAg.120-pIDKE2 plasmid interaction studies: In vitro protein-nucleic acids assembly reactions were carried out using conditions previously described ${ }^{[9,11]}$. Ten micromolar of purified $\mathrm{HCcAg} .120$ in renatured buffer was mixed with an equal volume of pIDKE2 plasmid at 100:1 protein-nucleic acid molar ratio. As control, denatured HCcAg.120 was resuspended in refolding buffer containing tRNA (Sigma) at 100:1 protein-nucleic acid molar ratio under RNase-free conditions. The reactions were incubated at $30{ }^{\circ} \mathrm{C}$ for $10 \mathrm{~min}$ followed by $15 \mathrm{~min}$ on ice and a $16,000 \mathrm{~g}$ spin at $4^{\circ} \mathrm{C}$ for $15 \mathrm{~min}$.

Equilibrium density gradient centrifugation: Five hundred microlitre of either renatured $\mathrm{HCcAg} .120$ or HCcAg.120-pIDKE2 plasmid samples were applied to a discontinuous $1-1.5 \mathrm{~g} \mathrm{~mL}^{-1}$ cesium chloride $(\mathrm{CsCl})$ density gradient in the same buffer of the protein sample, centrifuged at $100000 \mathrm{~g}$ for $26 \mathrm{~h}$ in a Beckmann SW40Ti rotor and fractionated. Five hundred microlitre aliquot of each fraction was collected from the bottom of the tube. Samples were also separated on a linear $25-70 \%$ sucrose density gradient solution and centrifuged at $100000 \mathrm{~g}$ for $16 \mathrm{~h}$. The refractive index of each fraction was measured using an Abbe-3L refractometer (Milton Roy). To detect HCcAg.120, fractions were assayed by Dot blot as previously reported ${ }^{[17]}$. The intensity of the resultant bands was quantified by measuring the optical density with the Eagle Eye II still video system (Stratagene).

Agarose gel electrophoresis: Samples containing either pIDKE2 plasmid or HCcAg.120-pIDKE2 plasmid were analyzed on agarose gel as previously described $^{[18]}$. The sample was mixed with $5 \mathrm{X}$ loading buffer (50\% glycerol, $0,02 \%$ bromophenol blue) and loaded onto a $1 \%$ agarose gel with ethidium bromide $\left(0.5 \mu \mathrm{g} \mathrm{mL}^{-1}\right)$. The gel was run in $1 \mathrm{X}$ TAE buffer and the gel was either detected under UV light or stained with Coomassie brilliant blue R250 (CBB, Sigma, St. Louis, USA).

Transmission electron microscopy (TEM): Either renatured HCcAg.120, or pIDKE2 plasmid, or HCcAg.120/pIDKE2 plasmid, or HCcAg.120/tRNA were fixed in glutaraldehyde and negatively stained with uranyl acetate prior to analysis by transmission electron microscopy as previously described ${ }^{[15]}$.

Detection of $\mathrm{HCV}$ core protein inhepatocytes: For Immunoelectron microscopy (IEM) analysis, samples of liver tissue from either $\mathrm{HCV}$-infected patients or healthy individuals ${ }^{[19]}$ were fixed with $4 \%(\mathrm{v} / \mathrm{v})$ paraformaldehyde containing $0.2 \%(\mathrm{v} / \mathrm{v})$ glutaraldehyde in $0.1 \mathrm{M}$ phosphate buffer $(\mathrm{pH} 7.3)$ at $4{ }^{\circ} \mathrm{C}$ for $3 \mathrm{~h}$ and washed with $0.1 \mathrm{M}$ phosphate buffer. Fixed cells were dehydrated as described above, embedded in Lowicryl and polymerized by exposure to ultraviolet light at room temperature for $72 \mathrm{~h}$. Ultrathin sections of liver biopsies were incubated with anti-HCcAg.120 IgG antibodies in phosphate buffer, for $45 \mathrm{~min}$ at RT. Specific reactions were detected as previously described $^{[19]}$. For immunofluorescence staining (IF) analysis, samples were immediately fixed with $4 \%$ paraformaldehyde in PBS at $4{ }^{\circ} \mathrm{C}$ and then mounted on gelatine-coated glass slides and stored for 2 days at $200{ }^{\circ} \mathrm{C}$. Then samples were treated as previously described ${ }^{[20]}$ and incubated overnight at $4{ }^{\circ} \mathrm{C}$ with with anti-HCcAg.120 IgG antibodies. Specific reactions were detected as described elsewhere ${ }^{[20]}$. Stained samples were coverslipped in Vectashield mountaing medium (Vector Laboratories, Inc. Burlingame, CA., USA), sealed with nail polish and viewed on an immunofluorescence microscope. 
Statistical analyses: All data were presented as mean \pm SE. Comparison of diameters of VLPs was conducted by Kruskal-Wallis test and Dunn's post test with GraphPad PRISM 4.0 software. $\mathrm{p}<0.05$ was considered statistically significant.

\section{RESULTS AND DISCUSSION}

Previous studies had demonstrated that $\mathrm{HCcAg}$ bound to the $60 \mathrm{~S}$ ribosomal subunit and RNA in vitro $^{[3,6,7,21]}$. In addition, Kunkel et al have suggested that interaction between $\mathrm{HCcAg}$ and nucleic acids in vitro plays a critical role for self-assembly of $\mathrm{HCcAg}$ into VLPs ${ }^{[9]}$. They also suggested that $\mathrm{HCcAg}$ can undergo extensive conformational changes upon binding to nucleic acid and assembly into $\operatorname{VLPs}^{[22]}$.

On the other hand, a previous study had demonstrated that a DNA vaccine containing the structural region of HCV (pIDKE2 plasmid) induces a strong humoral and cellular immune response in various animal models ${ }^{[12,13]}$. Since particulate antigens have been proposed as potential carriers for DNA vaccines ${ }^{[14]}$, the capacity of HCcAg.120 to interact with pIDKE2 plasmid was investigated.

HCcAg.120 was mixed with pIDKE2 plasmid at 100:1 protein-nucleic acid molar ratio as described above. Firstly, a density gradient centrifugation was used to demonstrate a possible interaction of pIDKE2 plasmid with HCcAg.120 in the VLPs assembly reactions. We expected that VLPs containing HCcAg.120 either in the presence or in the absence of pIDKE2 plasmid should differ in buoyant density according to their nucleic acids content.

As shown in Fig. 1, the $\mathrm{CsCl}$ gradient was fractionated and the individual fractions assayed for the presence of HCcAg.120. A peak fraction containing the renatured HCcAg.120 in the absence of pIDKE2 plasmid that migrated to a position in the gradient corresponding to a buoyant density of $1.15-1.17 \mathrm{~g} \mathrm{~mL}^{-1}$ was observed (Fig. 1A). Interestingly, the sedimentation behavior of HCcAg.120 changed from 1.15-1.17 $\mathrm{g} \mathrm{mL}^{-1}$ to $1.30-1.34 \mathrm{~g} \mathrm{~mL}^{-1}$ in HCcAg.120pIDKE2 plasmid samples, indicating a possible interaction between VLPs containing HCcAg.120 and pIDKE2 plasmid (Fig. 1B). Similarly, sucrose gradient analysis showed high density sucrose fractions containing $\mathrm{HCcAg}$ in $\mathrm{HCcAg}$.120-pIDKE2 plasmid samples (not shown).

These high density HCcAg.120 fractions were only observed in HCcAg.120-pIDKE2 plasmid samples. This is in agreement with previous results showing that native core-like particles containing nucleic acids obtained from recombinant E. coli and Pichia pastoris cells migrate with buoyant densities values ranging from $1.28 \mathrm{~g} \mathrm{~mL}^{-1}$ to $1.32 \mathrm{~g} \mathrm{~mL}^{-1}$ in $\mathrm{CsCl}$ and sucrose gradients ${ }^{[10,11,15]}$. It is interesting to note that native nucleocapsid particles from sera of $\mathrm{HCV}$-infected patients show high buoyant density values in sucrose

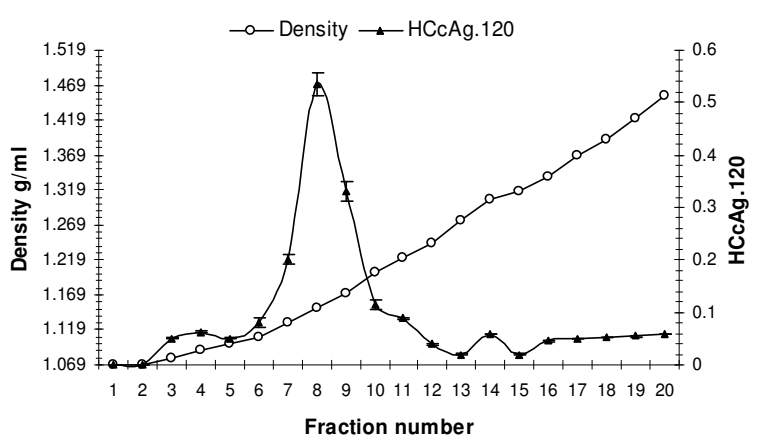

A

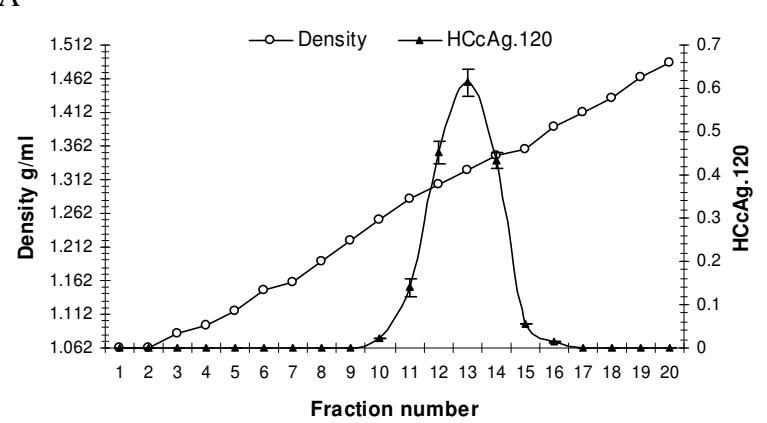

B

Fig. 1: $\mathrm{CsCl}$ gradient centrifugation analysis. The $\mathrm{CsCl}$ density of each fraction is shown (open circles; density (left ordinate) expressed in grams per milliliter). Detection of HCcAg.120 was analyzed by densitometry of dot blot (closed triangles; HCcAg. 120 (right ordinate) expressed as signal (OD) at $620 \mathrm{~nm}$ ). A) Analysis of renatured HCcAg.120. B) Analysis of HCcAg.120-pIDKE2 plasmid samples

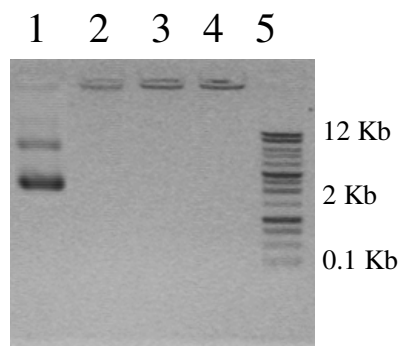

A

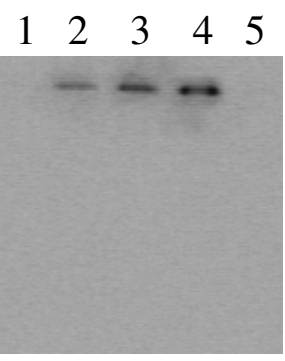

B
Fig. 2: Presence and characteristics of HCcAg.120-pIDKE2 from $\mathrm{CsCl}$ fractions (12-14). 1\% agarose gel of the HCcAg.120-pIDKE2 complexes visualized with ethidium bromide (A) and Comassie Blue (B). The lanes contain: pIDKE2 plasmid (1); $\mathrm{CsCl}$ fractions (12-14) containing HCcAg.120-pIDKE2 complexes (2-4, respectively); $1 \mathrm{~Kb}$ PlusLadder ${ }^{\mathrm{TM}}$ (Gibco BRL) size markers (5)

and $\mathrm{CsCl}$ gradients ${ }^{[4,23]}$. In addition, nucleocapsid particles obtained after detergent treatment from sera of chimpanzees and humans with chronic non-A, non-B hepatitis, have shown similar buoyant density values $^{[4,24]}$. Data suggest that samples containing HCcAg.120-pIDKE2 plasmid showed similar buoyant density values to those previously observed for nucleocapsids found in HCV-infected patients. 


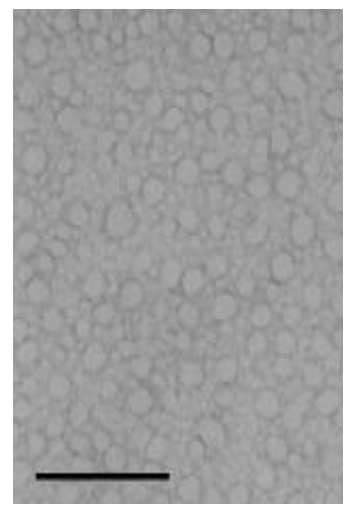

A

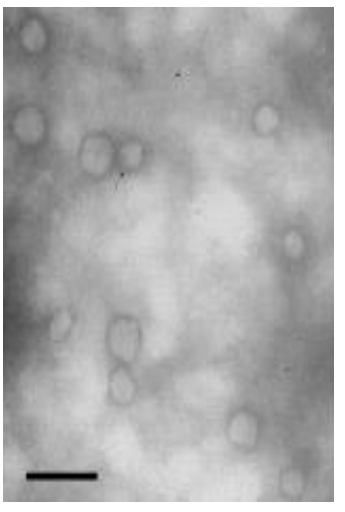

B

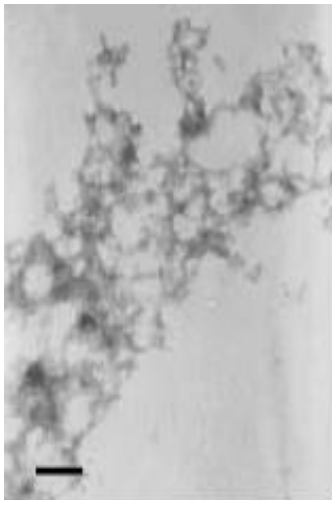

C

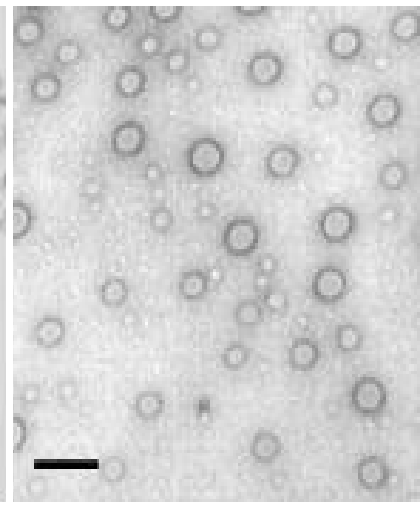

$\mathrm{D}$

Fig. 3: Transmission electron microscopy images of samples containing HCcAg.120. Micrograph of negatively stained samples containing: A) $\mathrm{CsCl}$ fractions (1.15$1.17 \mathrm{~g} \mathrm{~mL}^{-1}$ ) containing renatured HCcAg.120 B) HCcAg.120-pIDKE2 plasmid C) pIDKE2 plasmid D) HCcAg.120-tRNA. Bar $200 \mathrm{~nm}$

Afterwards, gradient fractions of HCcAg.120pIDKE2 plasmid samples were electrophoresed on $0.5 \%$ agarose gels and stained with $\mathrm{EtBr}$. The formation of HCcAg.120-pIDKE2 plasmid complexes are shown in Fig. 2. Intense EtBr staining was seen in all the cases. A delay in electrophoretic mobility of HCcAg.120pIDKE2 samples was observed (Fig. 2A). EtBr-stained bands were also positive for Coomasie staining, indicating that these were indeed HCcAg.120-pIDKE2 complexes (Fig. 2B).

In addition, assembly reactions containing HCcAg.120 were analyzed by TEM. Homogeneous particles with an average diameter of approximately 30 $\mathrm{nm}(29.91 \mathrm{~nm}, \mathrm{SD} 5,84)$ were observed for gradient fractions containing renatured HCcAg.120 (1.15-1.17 g $\mathrm{mL}^{-1}$ ), indicating that $\mathrm{CsCl}$ and sucrose gradient conditions did not affect the VLPs (Fig. 3A). Figure 4A shows the frequency distribution of particle diameters. The diameters of these particles ranged from 20 to 41 $\mathrm{nm}$. Remarkably, renatured HCcAg.120 (present in HCcAg.120-pIDKE2 plasmid samples) assembled into larger and heterogeneous VLPs (average diameter of $78.63 \mathrm{~nm}$, SD 29.59) (Fig. 3B and 4D).

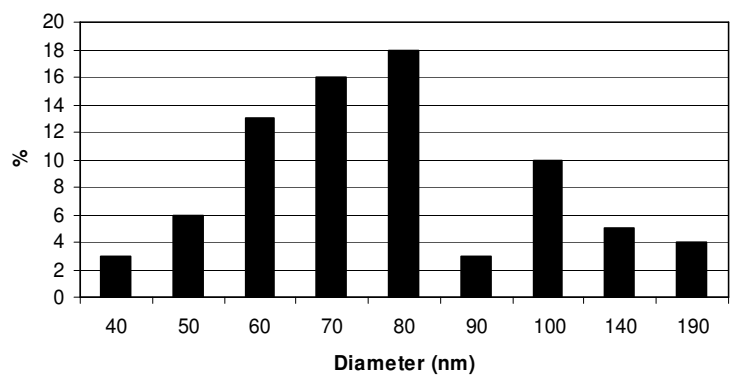

A

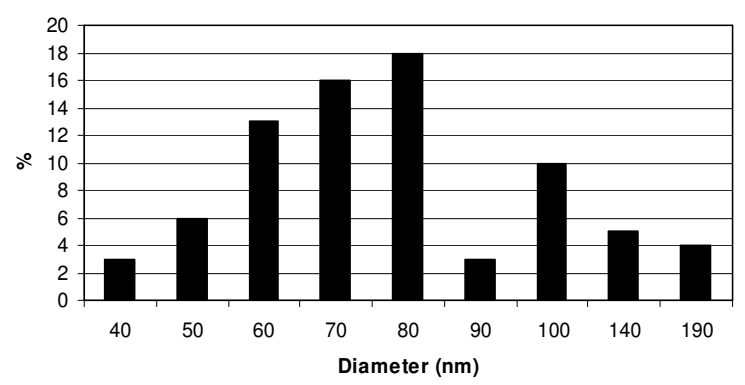

B

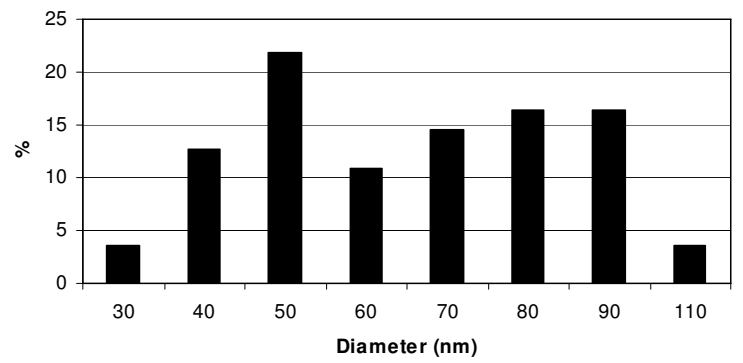

C

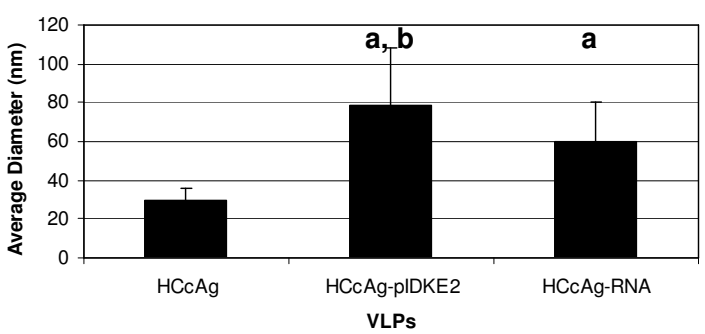

D

Fig. 4: Histogram representing the frequency distribution of particle diameters observed by TEM in the assembly experiments of $\mathrm{HCcAg} .120$. The diameter of particles was precisely measured in at least 10 fields (from 250 to 500 VLPs) from electron micrographs. Diameters of VLPs derived from renatured HCcAg.120 (A) or HCcAg.120-pIDKE2 plasmid (B) or HCcAg.120tRNA (C) are shown. (D) Comparison of diameters of VLPs from different in vitro assembly reactions containing HCcAg.120. (a) HCcAg-pIDKE2,HCcAgtRNA $>\mathrm{HCcAg}, \quad \mathrm{p}<0.001 ; \quad$ (b) $\mathrm{HCcAg}-$ pIDKE2 $>$ HCcAg-tRNA, $\mathrm{p}<0.05$

Figure 4B shows the frequency distribution of particle diameters. The diameters of these particles ranged from 36 to $190 \mathrm{~nm}$. However, these VLPs were not observed 


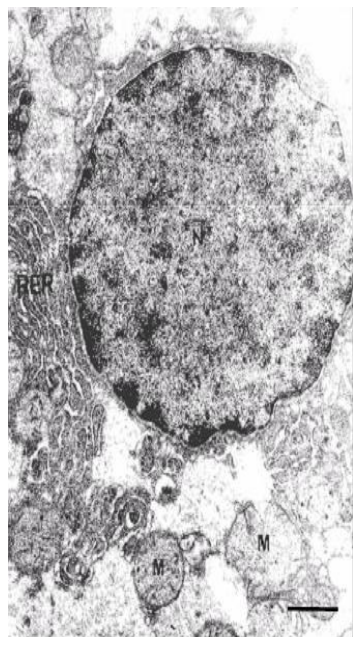

A

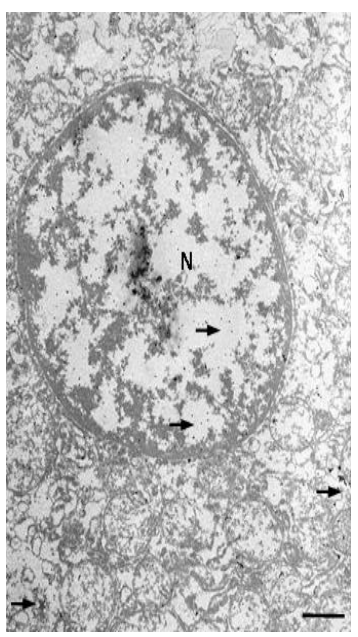

B

Fig. 5: Immunolabeling of $\mathrm{HCcAg}$ in hepatocytes of liver biopsies from HCV-infected patients by IEM. A) No immunostaining was observed in hepatocytes of liver biopsies from healthy individuals. B) Immunostaining (arrows) of liver sections from HCV-infected patients revealed $\mathrm{HCcAg}$ in cytoplasm and nucleus of hepatocytes. Nucleus (N); Mitochondria (M); Rough endoplasmic reticulum (RER). (Bar=1 $\mu \mathrm{m})$

in pIDKE2 plasmid samples (Fig. 3C). It is interesting to note that HCcAg.120 also assembled into heterogeneous VLPs in the presence of tRNA molecules (average diameter of $59.89 \mathrm{~nm}$, SD 20.16) (Fig. 3D and 4C). The diameters of these particles ranged from 25 to $100 \mathrm{~nm}$. These particles were larger than those obtained in HCcAg.120-only assembly reactions but shorter than those observed in HCcAg.120-pIDKE2 plasmid assembly reactions (Fig. 4D).

Finally, IEM (Fig. 5) and IF analysis (not shown) showed that anti-HCcAg.120 IgG antibodies, obtained in mice immunized with VLPs containing HCcAg.120 (not shown), speciffically reacted with hepatocytes from $\mathrm{HCV}$-infected patients but not with hepatocytes from healthy individuals. This suggest that these VLPs share epitopes with the $\mathrm{HCcAg}$ expressed during $\mathrm{HCV}$ infection in hepatocytes.

Previously, in vitro assembly of a C-terminal truncated HCcAg (covering the first 124 aa) into VLPs using a purified recombinant protein from $E$. coli was observed only in the presence of structured $\mathrm{RNA}^{[9]}$. These VLPs were heterogenous in size and larger than the expected native HCV nucleocapsids. HCcAg.120 assembled into homogeneous VLPs in the absence of structured nucleic acids. However, addition of either pIDKE2 plasmid or tRNA molecules to assembly reactions leaded to larger and heterogenous VLPs which appeared similar to those previously described ${ }^{[9]}$. One hypothesis is that the denaturation-renaturation process partially affected the folding of the protein which leads to the formation of larger agglomerates in the presence of nucleic acids. Indeed a recent report describes the purification of various variants of $\mathrm{HCcAg}$ under native conditions ${ }^{[11]}$. $\mathrm{HCcAg}$ assembled in vitro into VLPs with similar size and appearance to native $\mathrm{HCV}$ nucleocapsids in the presence of structured RNA. In addition, only the first $79 \mathrm{~N}$-terminal aa of $\mathrm{HCcAg}$ assembled in vitro into homogeneous particles of regular size and shape in the presence of structured tRNA. However, a few contaminants copurified with the recombinant proteins and their contribution to the VLPs assembly should be studied ${ }^{[11]}$.

Another hypothesis would be that nucleic acids misdirect in vitro assembly of VLPs. Misdirectors are postulated to disturb the geometry of intersubunit contacts and convert a growing spherical polymer, which will incorporate only a few hundred subunits, into a polymer that can incorporate an unlimited number ${ }^{[25,26]}$. A misdirector not only should prevent subunits from forming spherical particles but also could lead to the formation of regular nonnative structures $^{[25,26]}$. Thus, it is possible that misdirection of VLPs assembly by nucleic acids leads to large and heterogenous VLPs formation.

Therefore, it is not clear why $\mathrm{HCcAg}$ assembled in vitro in the presence of nucleic acids into particles larger than those isolated from infected subjects. In vivo, it has been suggested that $\mathrm{HCcAg}$ interaction with cell membranes or lipids and/or envelope glycoproteins may be required for correct viral particle morphogenesis ${ }^{[18,27]}$. So, it is possible that some cellular factors might be required for proper in vitro assembly of nucleocapsid-like particles.

\section{CONCLUSION}

Results presented in this work showed that interaction of HCcAg.120 with plasmid DNA and tRNA leads to in vitro assembly of heterogeneous VLPs. This approach would be important to elucidate the mechanisms and cellular factors that might be required for proper in vitro assembly of nucleocapsid-like particles. Besides, the capacity of particles containing HCcAg.120 to interact with nucleic acids could be used in the development of DNA vaccines and viral vectors based on these particles.

\section{ACKNOWLEDMENTS}

The authors thank Jesus Seone and Nilda Tamayo for their excellent technical assistance and Dr. Rafael F. Sanchez-Betancourt and Prof. Orlando J. Alvarez Guerrero for critical reading of the manuscript and for many helpful suggestions. 


\section{REFERENCES}

1. Choo, Q.L., G. Kuo, A.J. Weiner, L.R. Overby, D.W. Bradley and M. Houghton, 1989. Isolation of a cDNA clone derived from a blood-borne nonA, non-B viral hepatitis genome. Science, 244: 359-362.

2. Grakoui, A., C. Wychowski, C. Lin, S.M. Feinstone and C.M. Rice, 1993. Expression and identification of hepatitis $\mathrm{C}$ virus polyprotein cleavage products. J. Virol., 67: 1385-1395.

3. Santolini, E., G. Migliaccio and N. La Monica, 1994. Biosynthesis and biochemical properties of the hepatitis $\mathrm{C}$ virus core protein. J. Virol., 68: 3631-3641.

4. Maillard, P., K. Krawczynski, J. Nitkiewicz, C. Bronnert, M. Sidorkiewicz, P. Gounon, J. Dubuisson, G. Faure, R. Crainic and A. Budkowska, 2001. Nonenveloped nucleocapsids of hepatitis $\mathrm{C}$ virus in the serum of infected patients. J. Virol., 75: 8240-8250.

5. Matsumoto, M., S.B. Hwang, K.S. Jeng, N. Zhu and M.M. Lai, 1996. Homotypic interaction and multimerization of hepatitis $\mathrm{C}$ virus core protein. Virology, 218: 43-51.

6. Shimoike, T., S. Mimori, H. Tani, Y. Matsuura and T. Miyamura, 1999. Interaction of hepatitis C virus core protein with viral sense RNA and suppression of its translation. J. Virol., 73: 9718-9725.

7. Tanaka, Y., T. Shimoike, K. Ishii, R. Suzuki, T. Suzuki, H. Ushijima, Y. Matsuura and T. Miyamura, 2000. Selective binding of hepatitis C virus core protein to synthetic oligonucleotides corresponding to the 5 ' untranslated region of the viral genome. Virology, 270: 229-236.

8. Yan, B.S., M.H. Tam and W.J. Syu, 1998. Selfassociation of the C-terminal domain of the hepatitis-C virus core protein. Eur. J. Biochem., 258: 100-106.

9. Kunkel, M., M. Lorinczi, R. Rijnbrand, S.M. Lemon and S.J. Watowich, 2001. Self-assembly of nucleocapsid-like particles from recombinant hepatitis $\mathrm{C}$ virus core protein. J. Virol., 75: 2119-2129.

10. Lorenzo, L.J., S. Duenas-Carrera, V. Falcon, N. Acosta-Rivero, E. Gonzalez, M.C. de la Rosa, I. Menendez and J. Morales, 2001. Assembly of truncated $\mathrm{HCV}$ core antigen into virus-like particles in Escherichia coli. Biochem. Biophys. Res. Commun., 281: 962-965.

11. Majeau, N., V. Gagne, A. Boivin, M. Bolduc, J.A. Majeau, D. Ouellet and D. Leclerc, 2004. The Nterminal half of the core protein of hepatitis $\mathrm{C}$ virus is sufficient for nucleocapsid formation. J. Gen. Virol., 85: 971-981.
12. Duenas-Carrera, S., L. Alvarez-Lajonchere, J. Cesar Alvarez-Obregon, A. Perez, N. AcostaRivero, D.M. Vazquez, G. Martinez, A. Vina, D. Pichardo and J. Morales, 2002. Enhancement of the immune response generated against hepatitis $\mathrm{C}$ virus envelope proteins after DNA vaccination with polyprotein-encoding plasmids. Biotechnol. Appl. Biochem., 35: 205-212.

13. Duenas-Carrera, S., A. Vina, R. Martinez, L. Alvarez-Lajonchere, J.C. Alvarez-Obregon, J. Marante, A. Perez, O. Mosqueda, G. Martinez and J. Morales, 2004. Immunization with a DNA vaccine encoding the hepatitis-C-virus structural antigens elicits a specific immune response against the capsid and envelope proteins in rabbits and Macaca irus (crab-eating macaque monkeys). Biotechnol. Appl. Biochem., 39: 249-255.

14. Beyer, T., M. Herrmann, C. Reiser, W. Bertling and J. Hess, 2001. Bacterial carriers and virus-likeparticles as antigen delivery devices: role of dendritic cells in antigen presentation. Curr. Drug Targets. Infect. Disord., 1: 287-302.

15. Acosta-Rivero, N., J.C. Aguilar, A. Musacchio, V. Falcon, A. Vina, M.C. de la Rosa and J. Morales, 2001. Characterization of the HCV core virus-like particles produced in the methylotrophic yeast Pichia pastoris. Biochem. Biophys. Res. Commun., 287: 122-125.

16. Boulant, S., M. Becchi, F. Penin and J.P. Lavergne, 2003. Unusual multiple recoding events leading to alternative forms of hepatitis $\mathrm{C}$ virus core protein from genotype 1b. J. Biol. Chem., 278: 45785-45792.

17. Acosta-Rivero, N., A. Rodriguez, A. Musacchio, V. Falcon, V.M. Suarez, G. Martinez, I. Guerra, D. Paz-Lago, Y. Morera, M.C. de la Rosa, J. MoralesGrillo and S. Duenas-Carrera, 2004. In vitro assembly into virus-like particles is an intrinsic quality of Pichia pastoris derived HCV core protein. Biochem. Biophys. Res. Commun., 325: 68-74.

18. Acosta-Rivero, N., A. Rodriguez, A. Musacchio, V. Falcon, V.M. Suarez, L. Chavez, J. MoralesGrillo and S. Duenas-Carrera. 2004. Nucleic acid binding properties and intermediates of $\mathrm{HCV}$ core protein multimerization in Pichia pastoris. Biochem. Biophys. Res. Commun., 323: 926-931.

19. Falcon, V., N. Acosta-Rivero, G. Chinea, J. Gavilondo, M.C. de la Rosa, I. Menendez, S. Duenas-Carrera, A. Vina, W. Garcia, B. Gra, M. Noa, E. Reytor, M.T. Barcelo, F. Alvarez and J. Morales-Grillo, 2003. Ultrastructural evidences of $\mathrm{HCV}$ infection in hepatocytes of chronically HCVinfected patients. Biochem. Biophys. Res. Commun., 305: 1085-1090. 
20. Falcón, V., N. Acosta-Rivero, M. Shibayama, J. Luna-Munoz, M. Miranda-Sanchez, J. Gavilondo, M.C. de la Rosa, I. Menéndez, B. Gra, W. García, S. Dueñas-Carrera, J. Silva, G. Chinea, M. González-Bravo, F. Alvarez, J. Morales, J. Kouri and V. Tsutsumi, 2005. Detection of HCV components and pathological reactions in apoptotic hepatocytes from chronically HCV-infected patients. Am. J. Infect. Diseases, 1: 12-24.

21. Fan, Z., Q.R. Yang, J.S. Twu and A.H. Sherker, 1999. Specific in vitro association between the hepatitis $\mathrm{C}$ viral genome and core protein. J. Med. Virol., 59: 131-134.

22. Kunkel, M. and S.J. Watowich, 2002. Conformational changes accompanying selfassembly of the hepatitis $\mathrm{C}$ virus core protein. Virology, 294: 239-245.

23. Ishida, S., M. Kaito, M. Kohara, K. TsukiyamaKohora, N. Fujita, J. Ikoma, Y. Adachi and S. Watanabe, 2001. Hepatitis C virus core particle detected by immunoelectron microscopy and optical rotation technique. Hepatol. Res., 20: 335-347.
24. Takahashi, K., S. Kishimoto, H. Yoshizawa, H. Okamoto, A. Yoshikawa and S. Mishiro, 1992. p26 protein and 33-nm particle associated with nucleocapsid of hepatitis $\mathrm{C}$ virus recovered from the circulation of infected hosts. Virology, 191: 431-434.

25. Prevelige, P.E.Jr., 1998. Inhibiting virus-capsid assembly by altering the polymerisation pathway. Trends Biotechnol., 16: 61-65.

26. Zlotnick, A., P. Ceres, S. Singh and J.M. Johnson, 2002. A small molecule inhibits and misdirects assembly of hepatitis B virus capsids. J. Virol., 76: 4848-4854.

27. Blanchard, E., C. Hourioux, D. Brand, M. AitGoughoulte, A. Moreau, S. Trassard, P.Y. Sizaret, F. Dubois and P. Roingeard, 2003. Hepatitis C virus-like particle budding: Role of the core protein and importance of its Asp111. J. Virol., 77: 10131-10138. 